\title{
Predictive value of microvessel features for the clinical response to neoadjuvant chemotherapy in cervical squamous carcinoma and the associations with prognosis
}

\author{
Weili $\mathrm{Li}^{1 \#}$, Cong Liang ${ }^{1 \#}$, Ping Liu ${ }^{1 \#}$, Yingying $\mathrm{Qi}^{2 \#}$, Hong Shen ${ }^{3}$, Mingwei $\mathrm{Li}^{4}$, Chunlin Chen ${ }^{1}$ \\ ${ }^{1}$ Department of Obstetrics and Gynecology, Nanfang Hospital, Southern Medical University, Guangzhou, China; ${ }^{2}$ Department of Obstetrics and \\ Gynecology, the Fifth Affiliated Hospital of Guangzhou Medical University, Guangzhou, China; ${ }^{3}$ Department of Pathology, School of Basic Medical \\ Sciences, Southern Medical University, Guangzhou, China; ${ }^{4}$ Department of Obstetrics and Gynecology, the Jiangmen Central Hospital, Jiangmen, \\ China \\ Contributions: (I) Conception and design: W Li, C Liang, H Shen; (II) Administrative support: P Liu, C Chen; (III) Provision of study materials or \\ patients: M Li, C Chen; (IV) Collection and assembly of data: Y Qi, C Liang; (V) Data analysis and interpretation: C Liang; (VI) Manuscript writing: \\ All authors. (VII) Final approval of manuscript: All authors. \\ "These authors contributed equally to this work. \\ Correspondence to: Chunlin Chen, PhD. Department of Obstetrics and Gynecology, Nanfang Hospital, Southern Medical University, No. 1838, \\ Guangzhou Avenue, Guangzhou 510515, China. Email: ccl1@smu.edu.cn.
}

Background: Neoadjuvant chemotherapy (NACT) may help reduce the difficulty of surgery and eliminate tiny tumor metastatic foci. It has been reported that $69.4 \%$ to $95 \%$ of patients with cervical cancer respond to NACT. The aim of this study was to evaluate the value of microvessel features in the prediction of response of cervical carcinoma to NACT and its association with the prognosis.

Methods: In all, 106 patients (FIGO stage IB2 and IIA2) received NACT followed by radical hysterectomy. Pre-treatment biopsy specimens and post-treatment surgical specimens were stained by immunohistochemistry using CD31 and CD105 antibodies and were counted by quantitative stereology. The correlation between microvessel features [microvessel density (MVD) and volume density (Vv)] and the clinical response and prognosis were determined using the Mann-Whitney U-test and logistic multivariate analysis.

Results: Of the 106 patients, 74 (69.4\%) responded to NACT. The chemotherapeutic response was more favorable in patients with poor pathological grades and tumors larger than $5 \mathrm{~cm}(\mathrm{P}=0.047, \mathrm{P}<0.010)$. According to the pretreatment tumor size and the pathological grade, the patients were divided into six subgroups. In the subgroup of patients with a pretreatment tumor size $\geq 5 \mathrm{~cm}$ and tumors of moderate pathologic grade, the pre-NACT CD31-Vv was higher in NACT nonresponders than in responders $(\mathrm{P}=0.028)$. The area under the ROC curve for CD31- $\mathrm{V}_{\mathrm{v}}$ was 0.767 . In the logistic multivariate analysis, CD31-Vv was not an independent factor that affected the clinical response. The survival analysis showed that although higher post-treatment CD31-Vv was associated with a worse prognosis, Cox proportional hazards regression analysis did not indicate that microvessel features are prognostic predictors $(\mathrm{P}>0.05)$. Both pathological grade and invasive depth were independent predictors of survival.

Conclusions: Pre-treatment CD31-Vv could be a predictor of chemosensitivity in one specific subgroup (pre-treatment tumor size $\geq 5 \mathrm{~cm}$ and moderate pathological grade). Post-treatment CD31-Vv is associated with a worse prognosis but is not an independent factor for overall survival.

Keywords: Neoadjuvant chemotherapy (NACT); cervical cancer; microvessels; clinical response; prognosis

Submitted Aug 16, 2020. Accepted for publication Nov 20, 2020.

doi: $10.21037 /$ tcr-20-2741

View this article at: http://dx.doi.org/10.21037/tcr-20-2741 


\section{Introduction}

Cervical cancer is the fourth leading cause of cancerrelated deaths among women (1). Although the effect of neoadjuvant chemotherapy (NACT) before radical hysterectomy on long-term survival remains controversial, NACT may help reduce the difficulty of surgery and eliminate tiny tumor metastatic foci (2). Previous research has established that NACT responders have a significantly lower recurrence rate and longer tumor-free survival than NACT nonresponders (3). Similar long-term outcomes were noted among NACT nonresponders and the patients in the primary surgery group. Delaying surgery for NACT may mean that NACT nonresponders miss the optimal timing for surgery, which could delay the treatment process and increase medical expenses. It is reported that $69.4 \%$ to $95 \%$ of patients respond to NACT (4-9), and recent developments in the field of NACT have led to a renewed interest in NACT sensitivity.

Although extensive research has been performed on the relationship between the microvasculature and prognosis (10-18), no single study has focused on the value of microvessel features for the prediction of response to NACT among cervical cancer patients. Tumor growth and metastases require angiogenesis (19). Human papillomavirus (HPV), which is the leading pathogen that causes cervical cancer, is linked to angiogenesis through hypoxia-inducible factor 1a (HIF-1a) (20). Furthermore, because pretreatment CD105-labeled microvasculature predicts a clinical response to chemotherapy in patients with breast cancer (21), we hypothesized that microvessel features also predict the response of patients with cervical carcinoma to NACT.

The objective of this study was to determine whether microvessel features measured immunohistochemically and stereologically can predict the clinical response to NACT. In addition, the role of microvessel features in prognosis was also explored. We present the following article in accordance with the STARD reporting checklist (available at http://dx.doi.org/10.21037/tcr-20-2741).

\section{Methods}

\section{Patients}

From June 2007 to December 2016, 106 patients with 2009 International Federation of Gynecology and Obstetrics (FIGO) stage (22) IB2 or IIA2 squamous cervical cancer at 13 hospitals in mainland China were retrospectively studied. The inclusion criteria were as follows: (I) FIGO stage IB2 and IIA2; (II) no treatment history for cervical cancer before NACT including preoperative radiotherapy, hormone drugs, or antiangiogenic therapy; (III) availability of pre-treatment paraffin-embedded biopsy specimens, which could be used for immunohistochemistry, from the Department of Pathology at each hospital. The mean age of patients was 47 years (range, 22-66 years). This study was part of the Chinese Clinical Cervical Cancer (FOUR-C) Project, which was approved by the institutional ethics committee of Nanfang Hospital, Southern Medical University (NFEC-2017-135). The overall survival was determined as survival from the date of surgery to the last follow-up or the date of death. The study was conducted in accordance with the Declaration of Helsinki (as revised in 2013). This study was approved by the ethics committee of Nanfang Hospital, Southern Medical University (NFEC2017-135) and individual consent for this retrospective analysis was waived.

\section{Treatment}

All patients received 1 to 3 courses of platinum-based NACT at regular intervals every 21 days and radical hysterectomy (type C2) plus pelvic lymphadenectomy was performed 2 to 3 weeks after the final course of NACT. NACT regimens included cisplatin-based regimen (TP, paclitaxel + cisplatin; BP, cisplatin + bleomycin; PBV, BP + vincristine; FP, 5-fluorouracil + cisplatin; IP, Irinotecan + cisplatin), carboplatin-based regimen (TC, paclitaxel + plus carboplatin; BC, bleomycin + carboplatin; FC, 5 -fluorouracil + carboplatin), and other platinum-based regimens (paclitaxel or docetaxel + nedaplatin or oxaliplatin; IN, Irinotecan + nedaplatin) (Table 1).

Tumor size was measured in detail by gynecological physical examination, transvaginal ultrasonography, CT, MRI and pathologic examination. According to the RECIST criteria (23), patients who achieved a complete response (CR) or a partial response (PR) were classified as NACT responders, while patients with a stable response (SD) or progressive disease (PD) were classified as NACT nonresponders.

\section{Immunobistochemistry}

Paraffin sections $(4 \mu \mathrm{m})$ of biopsy specimens and surgical specimens were stained for CD31 and CD105. Paraffinembedded tumor specimens were deparaffinized in xylene and rehydrated in graded alcohol solutions. After they were 
Table 1 Clinical characteristics of the neoadjuvant chemotherapy responders and neoadjuvant chemotherapy nonresponders

\begin{tabular}{|c|c|c|c|c|c|}
\hline Variable & Subgroup & NACT responders $(n=74)$ & NACT nonresponders $(n=32)$ & $t / \chi^{2}$ & $P$ \\
\hline \multirow[t]{2}{*}{ FIGO stage } & IB2 & $51(73.9 \%)$ & $18(26.1 \%)$ & 1.578 & 0.209 \\
\hline & IIA2 & $23(62.2 \%)$ & $14(37.8 \%)$ & & \\
\hline \multirow[t]{2}{*}{ Pathological grade } & Good & $7(87.5 \%)^{a b}$ & $1(12.5 \%)$ & 10.208 & $0.006^{\dagger}$ \\
\hline & Poor & $43(81.1 \%)^{a}$ & $10(18.9 \%)$ & & \\
\hline \multirow[t]{2}{*}{ Tumor diameter } & $\geq 5 \mathrm{~cm}$ & $67(82.7 \%)$ & $14(17.3 \%)$ & 27.138 & $<0.01$ \\
\hline & $<5 \mathrm{~cm}$ & 7 (28.0\%) & $18(72.0 \%)$ & & \\
\hline NACT cycles & 1 & $42(66.7 \%)$ & $21(33.3 \%)$ & 1.125 & 0.771 \\
\hline \multirow[t]{3}{*}{ Regimen $^{\S}$} & Cisplatin-based & $42(76.4 \%)$ & $13(23.6 \%)$ & 2.329 & 0.312 \\
\hline & Carboplatin-based & $22(62.9 \%)$ & $13(37.1 \%)$ & & \\
\hline & Other platinum-based & $10(62.5 \%)$ & $6(37.5 \%)$ & & \\
\hline \multirow[t]{3}{*}{ Approach } & Arterial & $10(62.5 \%)$ & $6(37.5 \%)$ & 2.580 & 0.275 \\
\hline & Intravenous chemotherapy & $63(72.4 \%)$ & $24(27.6 \%)$ & & \\
\hline & Arterial+ Intravenous & $1(33.3 \%)$ & $2(66.7 \%)$ & & \\
\hline
\end{tabular}

${ }^{\dagger}$, Pairwise comparisons were performed for significant three-group comparisons. Significant differences in the table are denoted by letter designations (letters "a" and "b"). The values sharing the same letters are not significantly different; ", Cisplatin-based regimen: TP, paclitaxel $\left(135-175 \mathrm{mg} / \mathrm{m}^{2}\right.$, day 1$),+$ cisplatin $\left(50 \mathrm{mg} / \mathrm{m}^{2}\right) ; \mathrm{BP}$, cisplatin $\left(50 \mathrm{mg} / \mathrm{m}^{2}\right)+$ bleomycin $15 \mathrm{mg} ; \mathrm{PBV}, \mathrm{BP}+\mathrm{vincristine}\left(1 \mathrm{mg} / \mathrm{m}^{2}\right)$; FP, 5-fluorouracil (500 mg, days $1-3)+$ cisplatin $\left(50 \mathrm{mg} / \mathrm{m}^{2}\right.$, day 1$)$; IP, irinotecan $\left(80 \mathrm{mg} / \mathrm{m}^{2}\right.$, day 1$)+$ cisplatin $\left(60-70 \mathrm{mg} / \mathrm{m}^{2}\right)$. Carboplatinbased regimen: TC, paclitaxel (135-175 mg/m², day 1) + carboplatin (area under the concentration time curve, 3.0-5.0, day 1); BC, bleomycin $22.5 \mathrm{mg}+$ carboplatin (AUC 3.0-5.0, day 1); FC, 5-fluorouracil (500 mg, day 1-3) + carboplatin (AUC 3.0-5.0, day 1). Other platinum-based regimens: paclitaxel $\left(135-175 \mathrm{mg} / \mathrm{m}^{2}\right.$, day 1$)$ or docetaxel $\left(75 \mathrm{mg} / \mathrm{m}^{2}\right)+$ nedaplatin $\left(80 \mathrm{mg} / \mathrm{m}^{2}\right.$, day 1$)$ or oxaliplatin $\left(130 \mathrm{mg} / \mathrm{m}^{2}\right.$, day 1); IN, Irinotecan $\left(160 \mathrm{mg} / \mathrm{m}^{2}\right.$, day 1$)+$ nedaplatin $\left(80 \mathrm{mg} / \mathrm{m}^{2}\right.$, day 1). NACT, neoadjuvant chemotherapy; FIGO, International Federation of Gynecology and Obstetrics.

rinsed in deionized water and immersion in $3 \%$ hydrogen peroxide, the sections were submerged in citrate buffer $(\mathrm{pH} 6)$ and heated in a microwave oven for antigen retrieval. Sections were incubated overnight at $4{ }^{\circ} \mathrm{C}$ with primary rabbit monoclonal antibodies against CD31 (ab-134168; dilution, 1/300; Abcam, Cambridge, MA, USA) or CD105 (ab-169545; dilution, 1/300; Abcam, Cambridge, MA, USA). The sections were incubated with a polymer-linked secondary antibody (Shenda, Shenzhen, China) for $30 \mathrm{~min}$, which was followed by visualization with liquid DAB for $10 \mathrm{~min}$. The sections were counterstained in hematoxylin, dehydrated in graded alcohols solutions and cleared in xylene.

\section{Quantification of microvessel features: microvessel density (MVD) and volume density (Vv)}

To quantify the microvessels, each immunohistochemically stained section was digitally scanned using a microscope (Olympus BX51 JAPAN). For each tumor section, 3-5 high power fields (HPFs) were captured and assessed at a 200x magnification by two independent observers. The field area was equal to $0.6 \mathrm{~mm}^{2}$.

Image Pro-plus (IPP, version 4.0, Media Cybernetics, MD, USA) software was used as our image analysis system. IPP spatial calibration should be established for the microvascular morphological evaluation. This is 
A

2 Image-Pro Plus - NACT71A CL-CD105-2.jpg (1/1)

File Edit Acquire Enhance Process Measure Macro Window Help

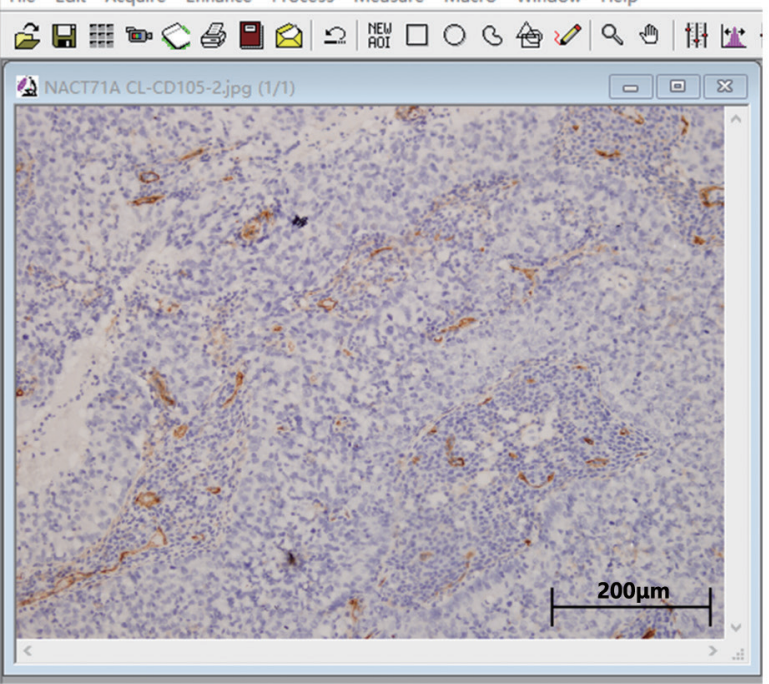

C

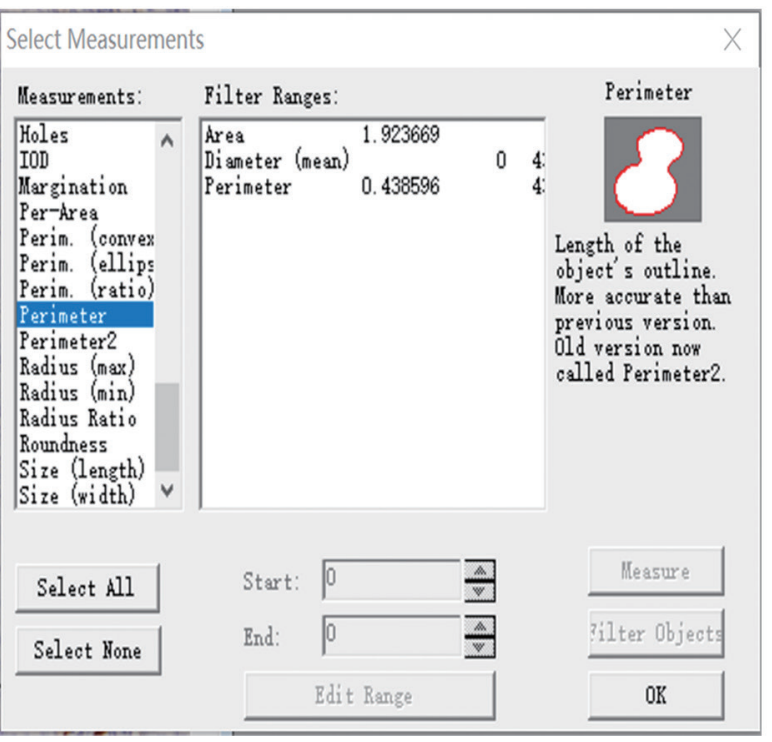

B

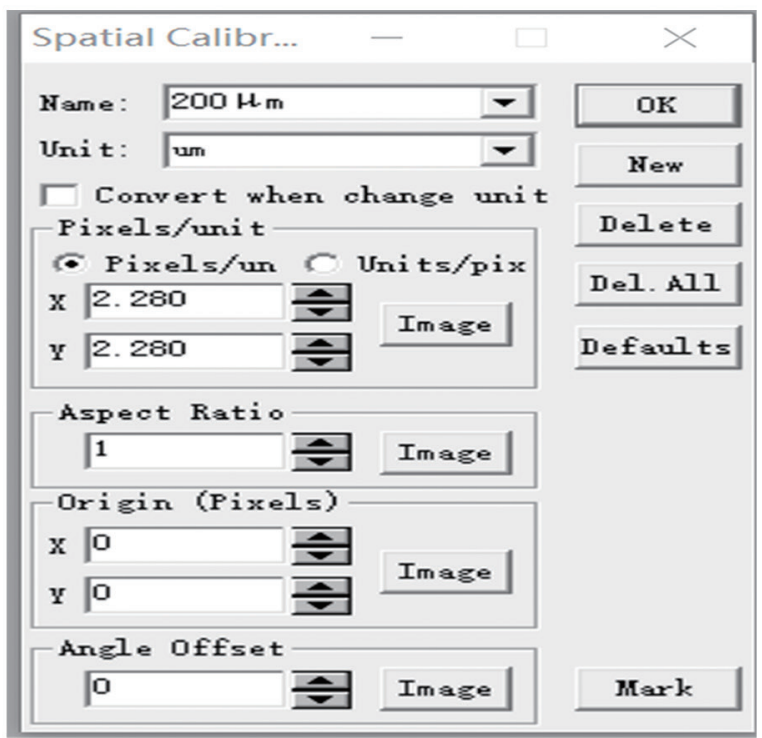

D

2. Image-Pro Plus - NACT85B-CD105-3.jpg (1/1)

File Edit Acquire Enhance Process Measure Macro Window Help

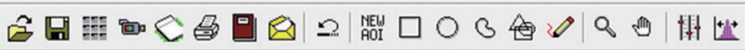

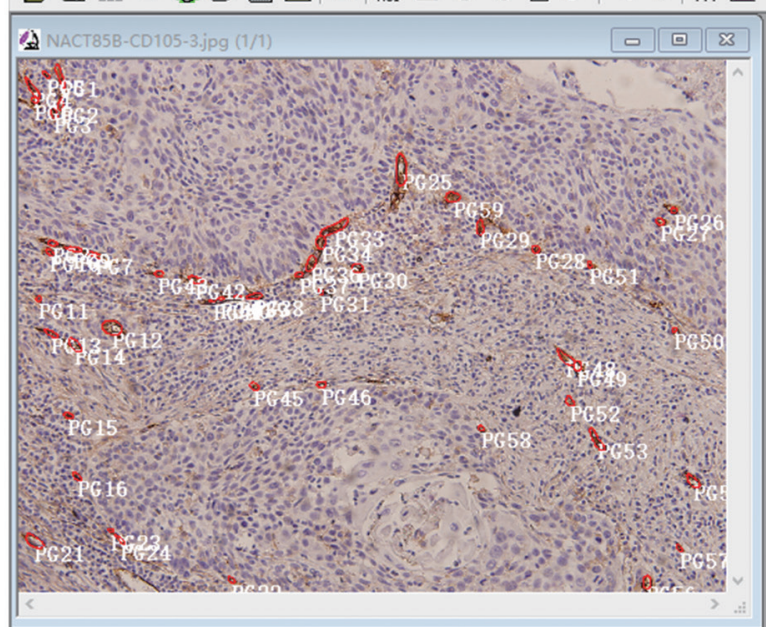

Figure 1 Stereological method for the measurement of microvessels in cervical cancer using Image Pro-plus software (IHC staining). (A) Import images; (B) spatial calibration; (C) select measurements; (D) microvessel assessment.

accomplished by manually outlining the microvessels with the cursor and then selecting the measurements "Area". The data regarding the object count and the area of each microvessel in the captured picture were exported to Excel (Figure 1).

$M V D=$ sum of object count/total images

$\mathrm{Vv}_{\mathrm{v}}=$ sum of object area/total area of the images

\section{Statistical analysis}

The statistical analysis was performed using SPSS 20.0 statistical software package (SPSS, Inc., Chicago, IL, USA). Normality was tested with the Kolmogorov-Smirnov test. Non-normally distributed variables are shown as medians $\left(\mathrm{P}_{25}-\mathrm{P}_{75}\right)$ and were evaluated with the MannWhitney $\mathrm{U}$ test. The chi-square test was used to compare 

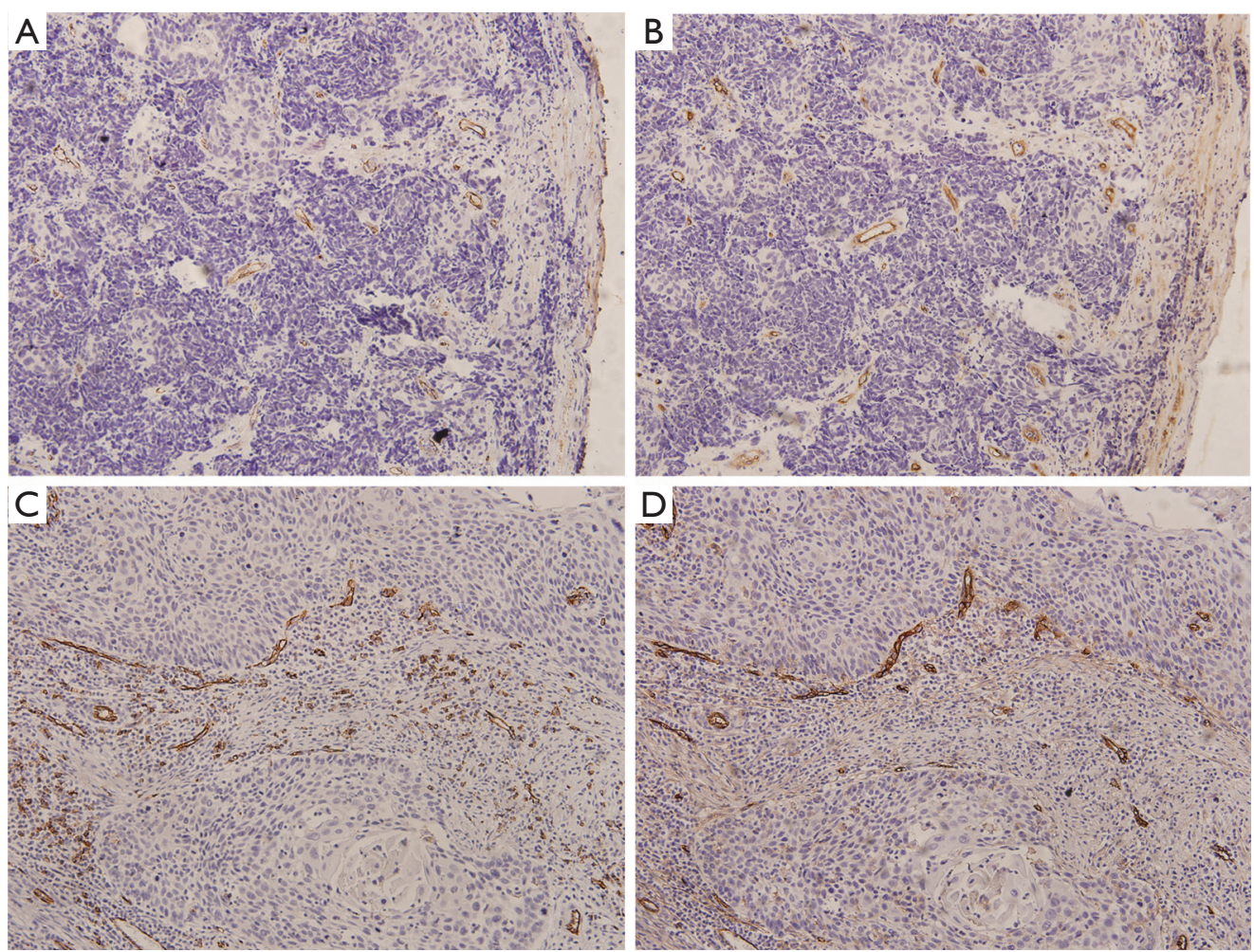

Figure 2 Immunohistological analysis of pre-neoadjuvant chemotherapy cervical cancer tissue sections $(\times 200)$. (A,B) Representative tissues of cervical cancer with poor pathological grade with positive staining for CD31 (A) and CD105 (B). (C,D) Representative tissues of cervical cancer with moderate pathological grade with positive staining for CD31 (C) and CD105 (D).

the categorical data. Logistic multivariate analysis was performed to determine the independent factors that are predictive of the response to NACT. In the survival analyses, the Kaplan-Meier method and log-rank test were used, and a multivariate analysis using the Cox proportional hazards model was also performed. The ROC curves were constructed and the area under the ROC curve (AUC) was calculated to evaluate the diagnostic accuracy. Youden's index was used to calculate the cut-off values. Statistical significance was defined as $\mathrm{P}<0.05$.

\section{Results}

\section{Clinical response to NACT and patient characteristic}

Representative examples of CD31 and CD105 positively stained microvessels in cervical cancer tissues are shown in Figure 2. According to the clinical chemotherapeutic response evaluation, 74 patients were classified as NACT responders $(74 / 106,69.8 \%)$, including 4 patients who achieved CR (3.8\%) and 70 who achieved PR (66.0\%);
32 patients were classified as NACT nonresponders, including 30 patients with SD $(28.3 \%)$, and 2 patients with PD (1.9\%). A higher clinical response rate was more frequent in patients with poor pathological grade and those with a tumor diameter $\geq 5 \mathrm{~cm}$. No significant difference was observed in other clinical characteristics, including age, FIGO stage, regimen cycles, chemotherapy drugs, or chemotherapy approaches between the two groups $(\mathrm{P}>0.05)$.

\section{Predictive value of pre-treatment microvessel features in terms of the clinical response}

Due to the uneven distribution of the two groups in terms of pathological grade and tumor diameter, the patients were divided into six subgroups. In the subgroup with pretreatment tumor size $\geq 5 \mathrm{~cm}$ and moderate pathological grade, pre-NACT CD31-Vv was higher in NACT nonresponders $(\mathrm{n}=8)$ than in NACT responders $(\mathrm{n}=22)$ $(\mathrm{Z}=-2.224, \mathrm{P}=0.026)$. In the other subgroups, no statistically significant difference was observed in the MVD or $\mathrm{Vv}$ 
Table 2 Comparison of MVD/ $\mathrm{Vv}$ in neoadjuvant chemotherapy responders and neoadjuvant chemotherapy nonresponders

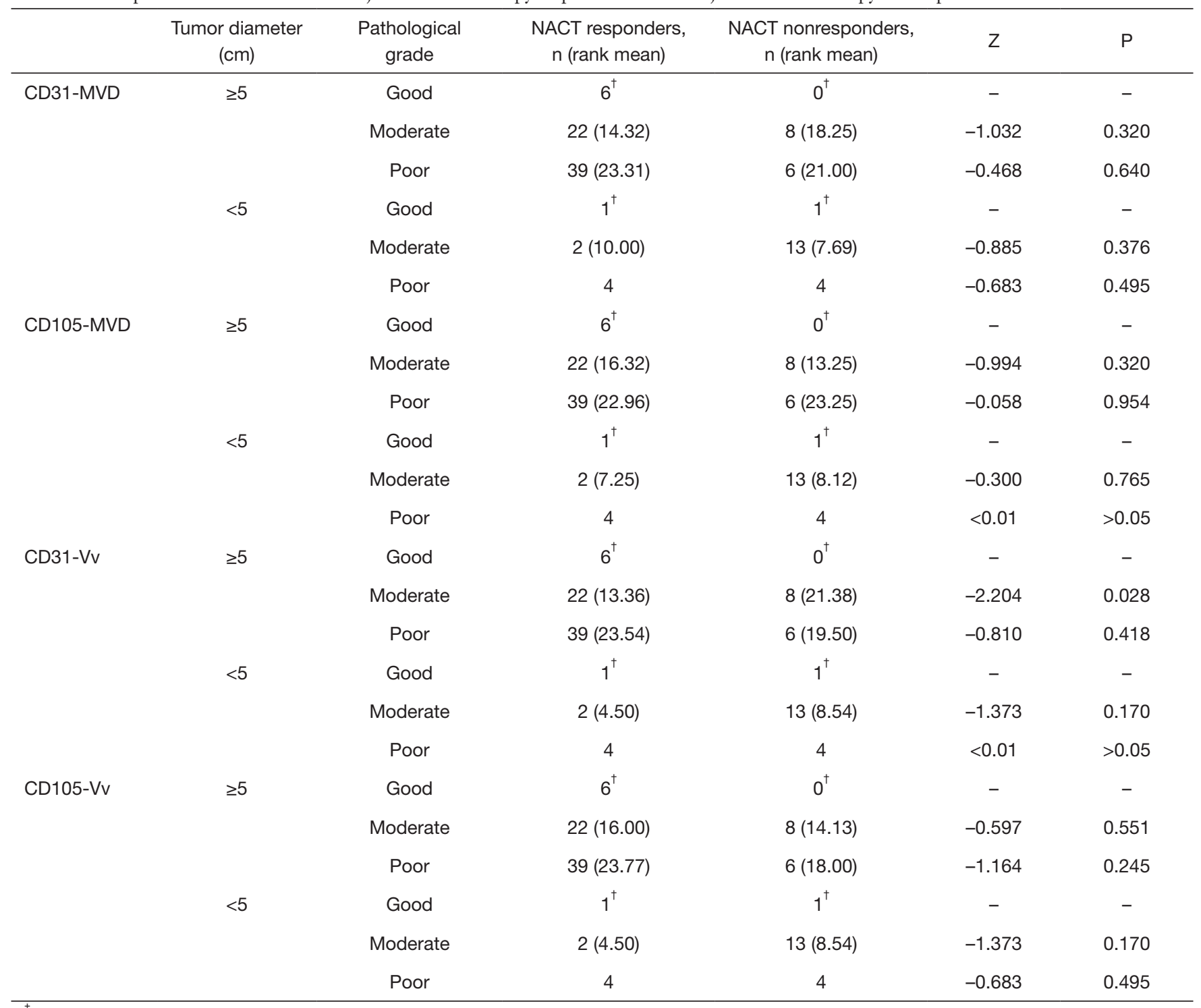

${ }^{\dagger}$, no statistical analysis due to the small number of cases. MVD, Microvessel density; Vv, volume density; NACT, neoadjuvant chemotherapy.

between the groups (Table 2).

The relationship between the diagnostic sensitivity and specificity is illustrated by the ROC curve (Figure 3). The ROC curve of the pre-NACT CD31- $\mathrm{V}_{\mathrm{v}}$ for predicting the chemotherapy response had an AUC value of 0.767 (95\% CI, 0.586 to $0.948, \mathrm{P}=0.028$ ) (Table 3). The optimal cutoff point was $5.691 \times 10^{-3}$, giving sensitivity of $59.10 \%$ and specificity of $87.5 \%$. In all, $87.50 \%$ of the patients who were not sensitive to chemotherapy could be identified using $\mathrm{CD} 31-\mathrm{Vv}_{\mathrm{v}}>5.691 \times 10^{-3}$, whereas $41.90 \%$ of patients who are sensitive were excluded.

The significant clinical factors from univariate analysis, as well as the chemotherapy approach, were entered into a multivariate analysis. Tumor size $\geq 5 \mathrm{~cm}(\mathrm{HR}=12.417$, $\mathrm{P}<0.01)$ and poor pathological grade $(\mathrm{HR}=0.297, \mathrm{P}=0.047)$ were independent factors that predicted the clinical response, while pre-NACT CD31- $\mathrm{Vv}_{\mathrm{v}}$ and chemotherapy approach were not predictive (Table 4).

\section{Survival}

Fourteen patients (9 in the NACT response group and 5 in the nonresponse group) died from cervical cancer. Two patients were excluded because they were lost to follow-up. 
Pathological grade and invasive depth were independent predictors of survival $(\mathrm{P}<0.05)$ (Table 5). Moreover, patients with a poor pathological grade and deep cervical stromal invasion had a significantly shorter overall survival.

The log-rank test showed that higher post-treatment $\mathrm{CD} 31-\mathrm{V}_{\mathrm{v}}$ was associated with a worse prognosis $(\mathrm{P}=0.032)$. However, microvessel features were not independent factors of overall survival in the multivariate analysis.

\section{Discussion}

The results of this study showed that pre-treatment CD31$\mathrm{V}_{\mathrm{v}}$ could be predictor of chemosensitivity in one specific subgroup (pretreatment tumor size $\geq 5 \mathrm{~cm}$ and moderate pathological grade). Post-treatment CD $31-V_{v}$ is associated with worse prognosis but is not an independent factor that affects prognosis.

Our research showed that the pre-NACT CD31$\mathrm{Vv}$ was higher in NACT nonresponders than in NACT

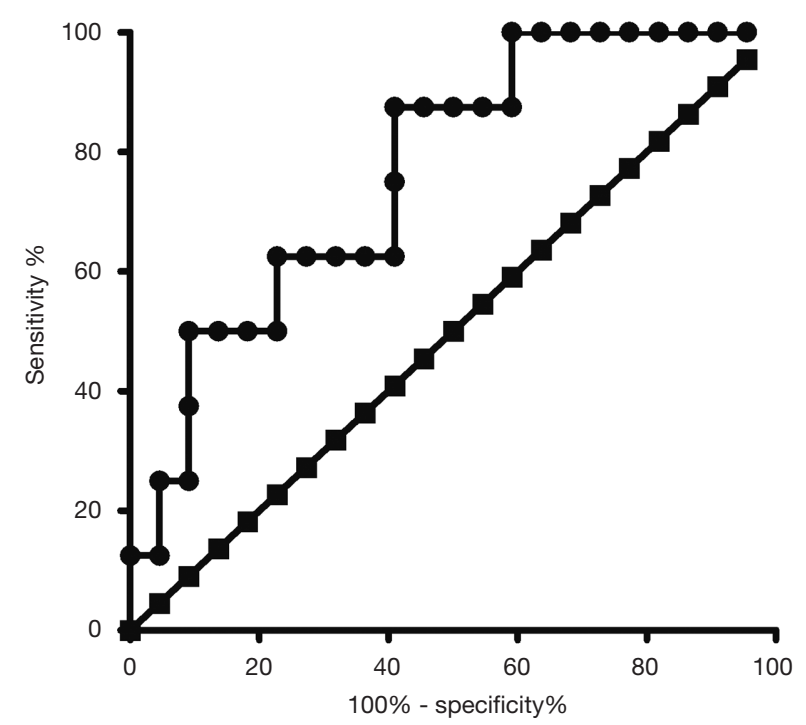

Figure 3 ROC curve of CD $31-V_{v}$ for the prediction of chemosensitivity in patient with a tumor size $\geq 5 \mathrm{~cm}$ and poor pathological grade. responders in the subgroup with pre-treatment tumor size $\geq 5 \mathrm{~cm}$ and moderate pathologic grade. The optimal cut-off point was $5.691 \times 10^{-3}$, giving sensitivity of $59.10 \%$ and a specificity of $87.5 \%$. This result demonstrates that $87.50 \%$ of patients who are not sensitive to chemotherapy can be identified using CD $31-\mathrm{Vv}_{\mathrm{v}}>5.691 \times 10^{-3}$. Therefore, if the patient meets the criteria of a specific subgroup criteria, NACT is not recommended if their pre-treatment biopsy CD31- $\mathrm{Vv}_{\mathrm{v}}$ is greater than the cut-off point. As a predictor, CD31- $\mathrm{V}_{\mathrm{v}}$ is only suitable for a portion of the patient population (maximum tumor diameter $\geq 5 \mathrm{~cm}$ and moderate differentiation). The sensitivity is only $59.10 \%$; that is, if the threshold is used as an indication of NACT, we would mistakenly exclude $40.9 \%$ of patients who might be sensitive to chemotherapy. While the ability to screen positive patients is weak, the ability to find true negative patients is strong. Since the effect of NACT followed by surgery on long-term survival remains controversial, the specificity which is the ability to screen negative patients, is more important in clinical practice. In addition, the relevant subgroup had a sample size of 30 . If we want to extend this conclusion to clinical applications, verification in larger external cohorts and the implementation of prospective clinical trials are required.

The prognostic value and the NACT sensitivity predictive value of differently labeled microvessels (CD31 and CD105) are distinctive. Although many studies have been performed to determine the relationship between the microvasculature and prognosis, they have presented contradictory findings (10-18). The results of these retrospective studies are likely influenced by the inclusion of women who received various medical treatments and those with varying tumor angiogenesis markers. Surveys such as those conducted by Randall et al. (18) have shown that CD31-MVD, but not CD105-MVD, is an independent prognostic factor for both PFS and OS in patients with high-risk, early-stage cervical cancer. CD105-MVD but not CD31-MVD or CD 34 MVD, was also identified to be a significant and independent predictor of recurrence in prostate cancer patients after radical prostatectomy with

Table 3 Area under the ROC curve (pre-neoadjuvant chemotherapy CD31-Vv)

\begin{tabular}{lccccc}
\hline \multirow{2}{*}{$\begin{array}{l}\text { Variable } \\
\text { CD31-Vv }\end{array}$} & Area & Standard error & $\mathrm{P}$ & \multicolumn{2}{c}{ 95\% Confidence interval } \\
\cline { 3 - 6 } & & & 0.028 & Lower limit & 0.586 \\
\hline
\end{tabular}

$\mathrm{Vv}$, volume density. 
Table 4 Results of multiple factor logistic regression analysis of clinical response

\begin{tabular}{lccc}
\hline Variables & Hazard ratio & 95\% Cl & P value \\
\hline Pathological grade & 0.297 & & 0.047 \\
Pathological grade 1 $^{\dagger}$ & 0.297 & $0.101-0.875$ & 0.028 \\
Pathological grade 2 $^{\dagger}$ & 1.852 & $0.154-22.205$ & 0.627 \\
Tumor diameter & 12.417 & $3.915-39.380$ & $<0.010$ \\
Pre-NACT CD31-Vv & 1.980 & $0.685-5.725$ & 0.207 \\
Chemotherapy & 1.520 & $0.382-6.051$ & 0.552 \\
approach & & & \\
\hline
\end{tabular}

${ }^{\dagger}$, Pathological grade 1: moderate vs. poor; Pathological grade 2: good vs. poor. NACT, neoadjuvant chemotherapy; Vv, Volume density.

neoadjuvant hormonal therapy (24). CD105-MVD but not CD31-MVD, is associated with metastasis-free survival and cause-specific survival in patients with urothelial cancer (25). This inverse prognostic value might imply the various biologic potentials of different tumor angiogenesis markers in different types of solid tumors.

Different angiogenesis markers have different efficacies in predicting the sensitivity of NACT. Beresford et al. (21) showed that pretreatment CD105 expression but not CD34 expression predicts for clinical response to chemotherapy, and that, a lower initial count is favorable. Our result is contrary to that reported in the studies by Beresford. The results of this study indicate that CD31-Vv but not CD105$\mathrm{V}_{\mathrm{v}}$ expression could be used as a predictor of clinical response. A possible explanation for this might be that different tumor tissues rely on different types of microvessels to provide oxygen and nutrition. Another possible explanation for this is that CD105-positive microvessels tend to be immature and irregularly shaped (26), while CD31-positive microvessels tend to be mature. Endoglin (CD105), a dimeric membrane glycoprotein that is expressed at high levels on human vascular endothelial cells, binds with high affinity to TGF $\beta 1$ and $\beta 3$ (27). CD105 has been found to be a specific marker of neoformed vessels. Therefore, we can deduce that these immature blood vessels cannot effectively transport chemotherapy drugs, which leads to a weak correlation with chemotherapy sensitivity. CD31 (platelet/endothelial cell adhesion molecule-1) supports the integrity of endothelial cell-cell junctions and leads to the over-expression of some protective genes that inhibit apoptosis $(28,29)$. It appears to antagonize chemotherapy-induced apoptosis. This may explain why patients with higher CD31-Vv are not sensitive to NACT. Preoperative chemotherapy plus angiogenesis treatment seems to result in better outcomes. Yang et al. (30) found that the baseline CD31 level is significantly associated with response to bevacizumab plus docetaxel-doxorubicin before surgery.

$V_{v}$ is more suitable than MVD for predicting chemosensitivity. In our study, CD31- $\mathrm{Vv}$, but not CD31MVD, could predict chemosensitivity in one specific subgroup. In the 1990s, Weidner et al. (31) proposed the use of MVD to quantify microvessels in tumors, and the average number of microvessels in 3 to 5 high-power fields was used as the MVD. In stereology, the length density refers to the length of a linear structure (such as a catheter, blood vessel, or microtubule) per unit volume. The length density is equal to the number of target cross-sections divided by the sum of the reference areas. The MVD is basically equivalent to the concept of length density in stereology. In the present study, the $\mathrm{Vv}$ of microvessels refers to the perfusion capacity of microvessels. MVD is more representative of parameters at the $2 \mathrm{D}$ level, while $\mathrm{Vv}$ is more representative of parameters at the $3 \mathrm{D}$ level.

In all patients, a multivariate logistic regression analysis of the clinical response showed that a tumor size $\geq 5 \mathrm{~cm}$ and poor pathological grade indicated a poor response to NACT but that the pre-treatment NACT CD31- $\mathrm{Vv}_{\mathrm{v}}$ and chemotherapy approach did not. This outcome is different than that of Chen et al. (3), who found that patients with tumors smaller than $8 \mathrm{~cm}$ had higher response rate, and that pathologic grade had no impact on chemotherapeutic response. The study by Chen $e t$ al. also mentioned that patients with good responses received a third cycle of chemotherapy, but they did not analyze whether the number of chemotherapy cycles influenced the clinical response. Our research revealed that patients with a tumor size less than $5 \mathrm{~cm}$ had a lower clinical response rate after NACT, and thus, selection bias may have occurred. For example, when a tumor shrinks from 4.5 to $3.5 \mathrm{~cm}$, it is reduced by $22 \%$, and when the tumor shrinks from 5.5 to $3.5 \mathrm{~cm}$, it is reduced by $36 \%$. Some gynecological oncologists believe that both post-treatment tumor sizes meet the same surgical requirements and that those patients do not need more cycles of chemotherapy. However, the difference in the proportion of decline determines the grouping according to efficacy, so that patients with tumor bigger than $5 \mathrm{~cm}$ are more likely to be categorized into chemotherapysensitive groups. In addition, patients $(81.1 \%, 43$ of 53$)$ with a poor pathological grade likely tended to achieve CR 
Table 5 Univariate and multivariate survival analyses for all patients who received neoadjuvant chemotherapy

\begin{tabular}{|c|c|c|c|c|}
\hline Factors & $\frac{\text { Univariate analysis }}{\mathrm{P} \text { value }}$ & \multicolumn{3}{|c|}{ Multivariate analysis (Cox's model) } \\
\hline Age & 0.647 & NS & - & - \\
\hline FIGO stage & 0.980 & NS & - & - \\
\hline Pathological grade & 0.018 & 0.032 & 4.091 & $1.132-14.781$ \\
\hline Lymph node metastasis & 0.038 & NS & - & - \\
\hline Parametrial infiltration & 0.752 & NS & - & - \\
\hline Margins & 0.616 & NS & - & - \\
\hline Invasive depth & $<0.001$ & 0.001 & 7.380 & $2.370-22.981$ \\
\hline Response to chemotherapy & 0.318 & NS & - & - \\
\hline Pretreatment CD31-MVD & 0.405 & NS & - & - \\
\hline Pretreatment CD105-MVD & 0.905 & NS & - & - \\
\hline Pretreatment CD31-Vv & 0.430 & NS & - & - \\
\hline Pretreatment CD105- Vv & 0.632 & NS & - & - \\
\hline Posttreatment CD31-MVD & 0.302 & NS & - & - \\
\hline Posttreatment CD105-MVD & 0.839 & NS & - & - \\
\hline Posttreatment CD31-Vv & 0.032 & NS & - & - \\
\hline
\end{tabular}

FIGO, International Federation of Gynecology and Obstetrics; LVSI, lymphovascular space invasion; MVD, Microvessel density; Vv, Volume density; NS, no significant; HR, hazard ratio; Cl, confidence interval.

or PR. Therefore, we are more concerned with the effects of vascular parameters on the response of patients with a moderate pathological grade.

However, in our study, most vascular parameters had no relationship with overall survival, regardless of whether the parameters were obtained before or after treatment. Although the log-rank test indicated that a higher posttreatment $\mathrm{CD} 31-\mathrm{Vv}$ was associated with a worse prognosis $(\mathrm{P}=0.032)$, a Cox hazard analysis did not indicate that the post-treatment $\mathrm{CD} 31-\mathrm{Vv}_{\mathrm{v}}$ was a prognostic predictor. Data from our study that are not presented here also showed that poor pathological grade was related to higher post-treatment CD31-Vv $(\mathrm{r}=-0.223, \mathrm{P}=0.023)$. Posttreatment CD31-Vv may have a collinear relationship with pathological grade, which may have led to the significant differences seen in the log-rank test. A seemingly similar result was found in pancreatic cancer. Wang et al. (32) reported that the tumor pathological grade was positively correlated with the MVD count in parenchymal cells of tumors, which suggests that the MVD is associated with the malignancy of pancreatic cancer.

Our study contributes to the current literature. Until now, scant attention has been paid to the relationship between the clinical response to NACT and microvascular features. First, NACT is generally considered an effective treatment for breast cancer and gastrointestinal tumors, but the role of NACT in the long-term efficacious treatment of cervical cancer is unclear. Second, due to the hierarchical healthcare system and pathological consultation process in China, most patients undergo cervical conization or colposcopy-directed biopsy in secondary medical institutions and then seek for surgical treatment at tertiary 
medical institutions, which means that paired biopsy specimens are rarely collected. Consequently, the literature on the relationship between the clinical response to NACT and microvascular features is sparse. Third, this study used quantitative stereology to evaluate microvascular volume.

We recognized several limitations in our study. First, the NACT regimens were not identical because we obtained the surgical specimens from multiple centers. Second, the time to recurrence in 14 patients with carcinoma-related death was difficult to obtain because their family member were unwilling to provider more detail. Therefore, we could not calculate the disease-free survival of those patients.

In conclusion, pre-treatment CD $31-\mathrm{Vv}_{\mathrm{v}}$ could be a predictor of chemosensitivity in one specific subgroup (pretreatment tumor size $\geq 5 \mathrm{~cm}$ and moderate pathological grade). Post-treatment CD31- $\mathrm{Vv}_{\mathrm{v}}$ is associated with a worse prognosis but is not an independent factor for overall survival.

\section{Acknowledgments}

We are grateful to En-Cheng Dai and Rui-Lei Liu (Department of Obstetrics and Gynecology, Linyi People's Hospital), Zhu-Mei Cui (Department of Obstetrics and Gynecology, The Affiliated Hospital of Qingdao University), Qian-Qing Wang (Department of Obstetrics and Gynecology, Central Hospital Affiliated to Xinxiang Medical University), Wei-Dong Zhao (Department of Gynecological Oncology, the First Affiliated hospital of USTC, Anhui Provincial Hospital), Min Hao (Department of Obstetrics and Gynecology, ShanXi Provincial cancer hospital), Pei-Yan Du (Department of Gynecological Oncology, Cancer Center of Guangzhou Medical University), Wu-Liang Wang (Department of Obstetrics and Gynecology, The Second Affiliated Hospital of Zhengzhou University), Long Chen (Department of Obstetrics and Gynecology, Qingdao Municipal Hospital), Shao-Guang Wang (Department of Obstetrics and Gynecology, Yantai Yuhuangding Hospital), and Ying Yang (Department of Obstetrics and Gynecology, Xinqiao Hospital) for their assistance with the specimens collection. We thank all participants in this study.

Funding: This work was supported by the National Science and Technology Support Program of China (2014BAI05B03), the Natural Science Fund of Guangdong Province (2015A030311024), the Medical Scientific Research Foundation of Guangdong Province of China (A2015063), and the Presidential Foundation of Nanfang
Hospital of Southern Medical University (2015C015). They provided financial assistance that played an important role in the collection of specimens.

\section{Footnote}

Reporting Checklist: The authors have completed the STARD reporting checklist. Available at http://dx.doi.org/10.21037/ tcr-20-2741

Data Sharing Statement: Available at http://dx.doi. org/10.21037/tcr-20-2741

Conflicts of Interest: All authors have completed the ICMJE uniform disclosure form (available at http://dx.doi. org/10.21037/tcr-20-2741). The authors have no conflicts of interest to declare.

Ethical Statement: The authors are accountable for all aspects of the work in ensuring that questions related to the accuracy or integrity of any part of the work are appropriately investigated and resolved. The study was conducted in accordance with the Declaration of Helsinki (as revised in 2013). This study was approved by the ethics committee of Nanfang Hospital, Southern Medical University (NFEC-2017-135) and individual consent for this retrospective analysis was waived.

Open Access Statement: This is an Open Access article distributed in accordance with the Creative Commons Attribution-NonCommercial-NoDerivs 4.0 International License (CC BY-NC-ND 4.0), which permits the noncommercial replication and distribution of the article with the strict proviso that no changes or edits are made and the original work is properly cited (including links to both the formal publication through the relevant DOI and the license). See: https://creativecommons.org/licenses/by-nc-nd/4.0/.

\section{References}

1. Bray F, Ferlay J, Soerjomataram I, et al. Global cancer statistics 2018: GLOBOCAN estimates of incidence and mortality worldwide for 36 cancers in 185 countries. CA Cancer J Clin 2018;68:394-424.

2. Rydzewska L, Tierney J, Vale CL, et al. Neoadjuvant chemotherapy plus surgery versus surgery for cervical cancer. Cochrane Database Syst Rev 2012;12:CD007406.

3. Chen $\mathrm{H}$, Liang $\mathrm{C}$, Zhang $\mathrm{L}$, et al. Clinical efficacy of 
modified preoperative neoadjuvant chemotherapy in the treatment of locally advanced (stage IB2 to IIB) cervical cancer: randomized study. Gynecol Oncol 2008;110:308-15.

4. Zanetta G, Lissoni A, Pellegrino A, et al. Neoadjuvant chemotherapy with cisplatin, ifosfamide and paclitaxel for locally advanced squamous-cell cervical cancer. Ann Oncol 1998;9:977-80.

5. Yamakawa Y, Fujimura M, Hidaka T, et al. Neoadjuvant intraarterial infusion chemotherapy in patients with stage IB2-IIIB cervical cancer. Gynecol Oncol 2000;77:264-70.

6. D'Agostino G, Distefano M, Greggi S, et al. Neoadjuvant treatment of locally advanced carcinoma of the uterine cervix with epirubicin, paclitaxel and cisplatin. Cancer Chemother Pharmacol 2002;49:256-60.

7. Dueñas-Gonzalez A, López-Graniel C, González-Enciso A, et al. A phase II study of multimodality treatment for locally advanced cervical cancer: neoadjuvant carboplatin and paclitaxel followed by radical hysterectomy and adjuvant cisplatin chemoradiation. Ann Oncol 2003;14:1278-84.

8. Cai HB, Chen HZ, Yin HH. Randomized study of preoperative chemotherapy versus primary surgery for stage IB cervical cancer. J Obstet Gynaecol Res 2006;32:315-23.

9. Choi CH, Kim T, Lee J, et al. Phase II study of neoadjuvant chemotherapy with mitomycin-c, vincristine and cisplatin (MVC) in patients with stages IB2-IIB cervical carcinoma. Gynecol Oncol 2007;104:64-9.

10. Rutgers JL, Mattox TF, Vargas MP. Angiogenesis in uterine cervical squamous cell carcinoma. Int J Gynecol Pathol 1995;14:114-8.

11. Bremer GL, Tiebosch ATMG, van der Putten HWHM, et al. Tumor angiogenesis: An independent prognostic parameter in cervical cancer. Am J Obstet Gynecol 1996;174:126-31.

12. Dellas A, Moch H, Schultheiss E, et al. Angiogenesis in cervical neoplasia: microvessel quantitation in precancerous lesions and invasive carcinomas with clinicopathological correlations. Gynecol Oncol 1997;67:27-33.

13. Obermair A, Wanner C, Bilgi S, et al. Tumor angiogenesis in stage IB cervical cancer: Correlation of microvessel density with survival. Am J Obstet Gynecol 1998;178:314-19.

14. Cooper RA, Wilks DP, Logue JP, et al. High tumor angiogenesis is associated with poorer survival in carcinoma of the cervix treated with radiotherapy. Clin Cancer Res 1998;4:2795-800.
15. Cooper RA, West CM, Wilks DP, et al. Tumour vascularity is a significant prognostic factor for cervix carcinoma treated with radiotherapy: independence from tumour radiosensitivity. Br J Cancer 1999;81:354-58.

16. West CM, Cooper RA, Loncaster JA, et al. Tumor vascularity: a histological measure of angiogenesis and hypoxia. Cancer Res 2001;61:2907-10.

17. Triratanachat $S$, Niruthisard S, Trivijitsilp $P$, et al. Angiogenesis in cervical intraepithelial neoplasia and earlystaged uterine cervical squamous cell carcinoma: clinical significance. Int J Gynecol Cancer 2006;16:575-80.

18. Randall LM, Monk BJ, Darcy KM, et al. Markers of angiogenesis in high-risk, early-stage cervical cancer: A Gynecologic Oncology Group study. Gynecol Oncol 2009;112:583-9.

19. Folkman J. What is the evidence that tumors are angiogenesis dependent? J Natl Cancer Inst 1990;82:4-6.

20. Bodily JM, Mehta KPM, Laimins LA. Human Papillomavirus E7 Enhances Hypoxia-Inducible Factor 1-Mediated Transcription by Inhibiting Binding of Histone Deacetylases. Cancer Res 2011;71:1187-95.

21. Beresford MJ, Harris AL, Ah-See M, et al. The relationship of the neo-angiogenic marker, endoglin, with response to neoadjuvant chemotherapy in breast cancer. $\mathrm{Br}$ J Cancer 2006;95:1683-8.

22. Pecorelli S. Revised FIGO staging for carcinoma of the vulva, cervix, and endometrium. Int J Gynaecol Obstet 2009; 105:103-4.

23. Eisenhauer EA, Therasse P, Bogaerts J, et al. New response evaluation criteria in solid tumours: Revised RECIST guideline (version 1.1). Eur J Cancer 2009;45:228-47.

24. Miyata Y, Mitsunari K, Asai A, et al. Pathological significance and prognostic role of microvessel density, evaluated using CD31, CD34, and CD105 in prostate cancer patients after radical prostatectomy with neoadjuvant therapy. Prostate 2015;75:84-91.

25. Miyata Y, Sagara Y, Watanabe S, et al. CD105 is a more appropriate marker for evaluating angiogenesis in urothelial cancer of the upper urinary tract than CD31 or CD34. Virchows Arch 2013;463:673-9.

26. Slevin M, Turu MM, Rovira N, et al. Identification of a 'Snapshot' of Co-Expressed Angiogenic Markers in LaserDissected Vessels from Unstable Carotid Plaques with Targeted Arrays. J Vasc Res 2010;47:323-35.

27. Cheifetz S, Bellon T, Cales C, et al. Endoglin is a component of the transforming growth factor-beta receptor system in human endothelial cells. J Biol Chem 1992;267:19027-30. 
28. Feng YM, Chen XH, Zhang X. Roles of PECAM-1 in cell function and disease progression. Eur Rev Med Pharmacol Sci 2016;20:4082-8.

29. Lertkiatmongkol P, Liao D, Mei H, et al. Endothelial functions of platelet/endothelial cell adhesion molecule-1 (CD31). Curr Opin Hematol 2016;23:253-9.

30. Yang SX, Steinberg SM, Nguyen D, et al. Gene Expression Profile and Angiogenic Markers Correlate with Response to Neoadjuvant Bevacizumab Followed by Bevacizumab

Cite this article as: Li W, Liang C, Liu P, Qi Y, Shen H, Li M, Chen C. Predictive value of microvessel features for the clinical response to neoadjuvant chemotherapy in cervical squamous carcinoma and the associations with prognosis. Transl Cancer Res 2021;10(1):162-173. doi: 10.21037/tcr-20-2741 plus Chemotherapy in Breast Cancer. Clin Cancer Res 2008;14:5893-9.

31. Weidner N, Semple JP, Welch WR, et al. Tumor angiogenesis and metastasis--correlation in invasive breast carcinoma. N Engl J Med 1991;324:1-8.

32. Wang SH, Sun YF, Liu Y, et al. CT contrast enhancement correlates with pathological grade and microvessel density of pancreatic cancer tissues. Int J Clin Exp Pathol 2015;8:5443-9. 